\title{
Menander und Josephos über Salmanassar IV.
}

\author{
Von C. F. Lehmann.
}

II. $\left.{ }^{1}\right)$

In dem alttestamentlichen Hauptbericht über Salmanassars IV: kriegerische Massnahmen gegen Hosea von Israël (2. Kön. 17, 1-6)²) endet der erste Feldzug Salmanassars gegen Hosea mit dessen Tributpflichtigkeit, genau wie bei Menander der gegen Elulaios mit Friedensschluss und Tributzahlung. Dann folgt der zweite Zug, bei dem Hosea, der im Vertrauen auf $\ddot{A} g y p t e n$ dem Assyrer den Gehorsam aufgesagt hat, alsbald gefangen in dessen Hand fällt, während Samaria erst nach dreijähriger Belagerung erobert wird. Hierzu stimmt wiederum aufs Beste Menanders Bericht über Salmancssars zweite, mehrjährige Expedition, die in die Belagerung von Tyrus ausläuft. Für die nevere alttestamentliche Kritik und Geschichtsschreibung ist diese Parallele nicht vorhanden, weil sie in völliger Abhängigkeit von der herrschenden irrigen Meinung Menander-Josephos' Bericht als für Salmanassar IV. ungültig betrachtet. So wird Salmanassars erster Zug mit Einstimmigkeit als ungeschichtlich übergangen.

Und auch von der danach verbleibenden einzigen Expedition glaubt man noch etwas abstreichen zu müssen. Während u. A. StaDE ${ }^{3}$ ) und W WLLHadSen ${ }^{4}$ ) ebenso wie TrEu: $^{5}$ ) mit der dem Fall Samarias erheblich vorausgehenden Gefangennahme Hoseas rechnen, wird diese Nachricht neuerdings für unhistorisch erklärt und durch die Behauptung ersetzt, Hosea sei beim Falle Samarias in der Assyrer Hände gefallen und mit dessen 27290 Bewohnern von Sargon weggeführt worden. Dabei wird seltsamerweise auch von dem letzten Bearbeiter der Inschriften Sargons $I I^{6}$.) ganz übersehen, dass die keilinschriftlichen Nachrichten über Samarias Fall in diesem Punkte eine direkte Bestätigung für 2. Könige 17 ergeben. Sargons einschlägige Berichte sind zwar mehrfach verstümmelt, wir haben aber auch wohlerhaltene Stellen: in keiner derselben wird erwähnt, dass der König unter den Gefangenen ge-

1) Vgl. oben S. 125/140. - 2) Vgl. 2. Kön. 18, 9-12, worüber unten. - 3) STade, Gesch. des Volkes Israel, S. $600^{1}$. - 4) Israel. u. jüd. Geschichte S. 81. - 5) Babyl.assyr. Gesch. S. 234. Vgl. o. S. 1281. - 6) Wisckrer, s. Flelmolts Weltgesch. III 203, vgl. die folgende Anmerkung. 
wesen wäre. $\left.{ }^{1}\right)$ Wer den Stil der assyrischen Königsinschriften einigermassen kennt, weiss, dass es dafür nur die eine Erklärung giebt, dass Sargon nicht mit einem Könige von Israel und Samaria zu thun gehabt hat. Und wer den assyrischen Quellen ferner steht, lese in einer Übersetzung die Zeilen, die der Erwähnung von Samarias Falle vorausgehen und folgen, und überzeuge sich, dass, wo immer Könige und Fürsten in den bekämpften Gebieten vorhanden waren, sie auch genannt werden, selbst wenn sie ihre Person in Sicherheit zu bringen wussten, wie Jaman von Asdod, der sich unter ägyptischen Schutz begab. Dass Hosea, wahrscheinlich gleich im Jahre 724, gefangen genommen wurde, steht also durch das Zengnis des Königsbuches und das Schweigen Sargons ebenso sicher, wie es aus den Zeitverhältnissen begreiflich ist.

Die Vorgänge in Phönizien liefern eine wertvolle und wirksame Ergänzung unserer Anschauungen. Zul Salmanassars zweitem Zug gegen Tyrus gab der Ruf der phönizischen Städte, die zu Assyrien hielten, Anlass. Eine assyrisierende Partei war auch in Israël vorhanden. Gerade als deren Haupt war ja Hosea, der Mörder des Pelah, von Tiglatpileser III. 734 als König über das freilich schon sehr beschnittene und geschwächte Reich. Samaria gesetzt worden. Dadurch dass Hosea sich mit Ägypten einliess, ergab sich eine Verschiebung der Parteiverhältnisse. Vermutlich standen den Anhängern der Assyrer deren Gegner nicht etwa geschlossen und einhellig gegenüber, sondern neben denen, die zu Ägypten hielten, ${ }^{2}$ ) wird, wie später in Juda zur Zeit

1) Verstummelt: Annalen 11; erbalten: Prunkinschr. 23; Ann. des Saales XIV 15; vgl. Pavé des portes IV 31 (Winckler, Keilschrifttexte Sargons Bd. 1).

2) Ein Hinundherschwanken zwischen Assyrien und Agypten, wie es gerade für den König Hosea historisch feststeht, wird wiederholt vom Propheten Hosea gegeisselt, der nach den einleitenden Worten des Buches Hosea von Jerobeam II. bis in die Zeit Histias, der mit Hosea gleichzeitig über Juda regierte, gelebt hat. Die herrschende Meinung hält diese Angabe für den irrigen Zusatz eines Redaktors. Da sich keine Hindentung auf das aramäisch-israelitische Bündnis gegen Juda finde, so gehöre die Prophetie Hoseas in die Zeit vor 738 v. Chr. (Cornrtr, Kautzscr). Dies argumentum ex silentio steht m. E. auf sehr schwachen Füssen. Der erste Teil des Buches Hosea Kap. 1-3, in dem der Prophet "sein häusliches Ungliuck berichtet und wie eben dies die Prophetie in ihm geboren hat" (CorntLx, Einl. \$23), gehört in die Zeit Jerobeams II. Kap. 4-14 „bilden wohl ein zusammenhängendes Resumé der prophetischen Predigt Hoseas, gegen Ende seiner Wirksamkeit von ihm selbst niedergeschrieben ". Wenn diese Prophezeiungen $\mathrm{nach} 738$ ergangen sind, in den Jahren des Niedergangs und der Vernichtung Israëls, so brauchte Hosea keineswegs auf die erledigten Angelegenheiten der Vorjahre zurückzugreifen. Nur von König Hosea wissen wir, dass er Anknüpfung an Ägypten gesucht und gefanden hat.r Nun soll freilich der Prophet Hosea von der 734 erfolgten Abtrennung des Ostjordanlandes nichts gewusst haben und deshalb nicht mehr unter seinem königlichen Namensvetter gewirkt resp. seine Prophezeiungen niedergeschrieben haben können. Aber gegenüber einer Stelle wie Hosea 12, 12 (vgl. $12,15)$ kanu diese Voraussetzung schwerlich aufrecht erbalten werden. Mit Winckuen, Helmolts Weltgesch. III 203 halte ich daher für wahrscheinlich, dass die prophetische 
Nebukadnezars und Jesajas, eine radikale, zumeist durch die Priesterschaft bestimmte Unabhängigkeitspartei gestanden haben, die überhaupt von einer fremden Einmischung, woher sie immer kommen mochte, nichts wissen wollte. Dass bei Hoseas Gefangennahme die von ihm verlassenen Anhänger Assyriens die Hand im Spiele hatten, sei es, dass sie ihn direkt auslieferten, sei es, dass er, bei etwaigen Zusammenstössen mit den Assyrern nur laue Unterstïtzung bei ihnen fand, ist wohl denkbar und mir persönlich sogar wahrscheinlich. Mit Hoseas Person geriet auch der bisher nicht annektierte Rest israëlitischen Gebietes in die Hände Salmanassars. Nur die Hauptstadt, in der die Gegner der Assyrer, und unter ihnen vermutlich wieder die Unabhängigkeitspartei, den Ton angaben, leistete weitere drei Jahre verzweifelten Widerstand. So hat es richtig anch WeLlHausen dargestellt.

Dass nun Hosea mit den Hilfsmitteln seiner geschwächten Herrscliaft den Widerstand gegen Salmanassar nicht allein hätte beginnen und organisieren können, liegt auf der Hand. Er stand offenbar mit Lulî von Tyrus im Bunde, und beide verliessen sich auf Ägypten. Mag auch die Identifikation des als Bundesgenossen Hoseas genannten Sewe' Schwierigkeiten machen, die übrigens keineswegs unüberwindlich sind $^{1}$ ): sicher ist dass mit dem Misrajim als dessen König Sewe' 2. K. 17 genannt wird, in diesem Falle nur Agypten gemeint sein kann. Gegenüber den neneren Versuchen ${ }^{2}$ ), Misrajim hier mit einem nordarabischen Staat zu identifizieren, der mit Ägypten nur den Namen gemein habe, muss dies nachdrücklich betont werden. Der Besitz der östlichen Mittelmeerküste und ilıres Hinterlandes ist zu allen Zeiten eine Lebensfrage für jedes im Zweistromlande wie im Niltal sich entwickelnde selbständige Staatswesen mit politisch und kommerziell expansiver Tendenz gewesen. Seit der Thutmosiden-Zeit besteht zwischen Ägypten und dem Zweistromland ein unablässiges, nicht immer offenkundiges, darum aber nicht minder nachhaltiges Ringen um diese Gebiete, das auch für die Zeiten der assyrischen Obmacht fortdanert. Als Tiglatpileser I. um $1000 \mathrm{v}$. Chr. ${ }^{3}$ ) an der nordphönikischen Küste erscheint, werden ihm Geschenke, u. A. ein Krokodil vom König von Musri (= Ägypten) gesandt. Wenn die Assyrerkönige u.A.Tiglatpileser I.(?) und Assurnasirabal neben den Siegestafeln Ramses' II. ihre Inschriften am Hundstlusse in den Felsen meisseln lassen, so geben sie dadurch zu erkennen, dass sie sich als Nachfolger der Ägypter in den Herrschaftsansprüchen über das Westland tühlen. Šešonq (22. Dyn.) ergreift nach Salomos Tode die Gelegenheit, sich in die Verhältnisse des zu gefährlicher Machtentwicklung gelangten Gesammtreiches Juda einzumischen. Tiglatpileser III. hatte

Wirksamkeit Hoseas, deren Niederschlag Kap.4-14 sind, in die Zeit nach der Eroberung von Damaskus fällt, natürlich ohne mich Wincklérs Deutung von Misrajim auf ein arabisches Mușri anzuschliessen, s. S. $469^{2}$.

1) S. u. S. 469 Anm. 2. - 2) Winckler, zuletzt in $K A T^{3} 136 \mathrm{ff}$ - 3) C. F. Lehmans, Zwei Hauptprobl. d. altor. Chronol. S. 99, 168/70. 
734 Damaskus, das letzte Bollwerk des Aramäertums, Assyrien einverleibt und ebenso Israël links des Jordan eingezogen. Vom Nillande war Assyriens Machtbereich nur noch durch Juda und die philistäische Pentapolis getrennt. Ägyptens Existenz war bedroht, - wie schwer, hat die nächste Folgezeit gelehrt: ein halbes Jahrhundert später war Ägypten assyrische Provinz. Ein Eingreifen Agyptens in diesem Augenblick müssten wir fordern und vermuten, wenn es nicht berichtet wäre. Dem Bestreben, den vorhandenen Bericht auf ein arabisches Musri umzudeuten, ist von vornherein das Urteil gesprochen. $\left.{ }^{1}\right)^{\circ}$

Wie nun die von Salmanassar begonnenen Unternehmungen gegen Israël und Tyrus, so spinnen sich auch, ganz wie zu erwarten, die Verwicklungen mit Ägypten unter Sargons II. Regierung fort.

Auch weiter im Süden und hier vor allem musste Ägypten suchen, den Assyrern thatkräftige und wagemutige Gegner zu erwecken. Sargon fand, wie später Alexander noch nach Tyrus' Falle, in Gaza energischen Widerstand, und eben den Sewe, mit dem Hosea und Elulaios angeknüpft hatten, treffen wir bei Sagon, der ihn Sib-'e nennt, ${ }^{2}$ ) als ägyptischen Verbündeten Hannos von Gaza.

1) Ich beschränke mich auf den Nachweis, dass an den uns hier angehenden Stellen des AT. Misrajim nur Ägypten bezeichnen kann. Die Frage, ob es ausser Musri-Ägypten und ausser dem kilikischen Gebiet gleichen Namens (Winckier, Altt. Untersuch. 172/4) iu berh a u $t$ ein arabisches Musri gegeben $172 / 4$ habe, lasse ich hier unerörtert. Doch vgl. d. folg. Anm.

2) Sewe'-Sib'e, 2 K. 17 König v. Ägypten, bei Sargon Turtan (Oberbefehlshaber) des Pir $u$ v. Muşur-Agypten kann, sprachlich und chronologisch, nicht mit Sabalko (assyr. $\check{S} a b a k \hat{u})$ identifiziert werden. Sein $\mathrm{Name}$ stimmt zu dem von Sabakos Sohne $\Sigma \varepsilon \beta \iota x$ ós (Kurzform[?] von $\check{S} a b a t a k a)$, Identität der Person ist nat. undenkbar. In der Dynastie Šabako's ist die Wiederholung von Namen aus der 1. in der dritten Generation uiblich.

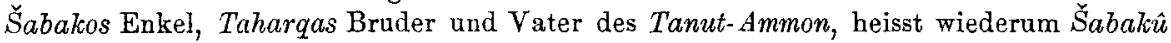
(Assurban., vgl. Steindonf, Beitr. z. Assyr. I 341). Ich balte Sib'e für einen Angehörigen der $\breve{S} a b a k o$ vorausgehenden Generation, etwa dessen Oheim, - ك̌abako's Vater hiess bekanntlich Kašto - , der unter Bok-n-ranf-Bolchoris bereits eine bedeutende Stellung als Unterkönig von Äthiopien eingenommen hat und deshalb, da Bokchoris die Initiative in den kriegerischen Angelegenheiten dem Athiopen überliess, im AT. mit einem gewissen Recht als König bezeichnet wird, während bei Sargon (722 v. Chr.), der staatsrechtlichen Sachlage genauer entsprechend, der Äthiope als Oberfeldherr, als erster Beamter nach dem Pharao, behandelt wird. Daraus, dass Bokchoris auf sein Oberkönigtum als Pharao besonderen Nachdruck legte, mag es sich auch z. T. erklären, dass er bei Sargon schlechtweg Pir'u = Pharao beisst Im Jahre 713 (s. Marquart, Chronol. Unters. S. 715 [71]) vollendete Šabako, was Pianchi I (vgl. dessen Verbältnis zu Tefnacht) und dessen Nachfolger auf dem ätbiop. Thron angebahnt hatten: Bokchoris wurde entthront und verbrannt, und Ägypten stand unter äthiop. Herrschaft. Dass sich nach 713 v. Chr. Sargons Terminologie ändert, ist eine weitere Bestätigung für meine Auffassung. Jaman von Asdod flieht nach Ägypten (Musri), ${ }_{n}$ das zu Äthiopien (MLeluhha $a^{4}=$ Meroë Jensen; im

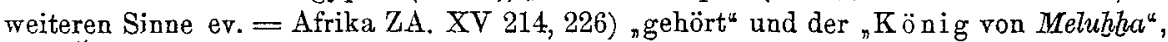
eben $\breve{S} a b a k o$ liefert ihn aus, offenbar weil er sich einer assyr. Invasion nicht gewachsen fühlt.

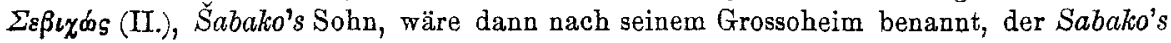


Soviel über den z weiten vulgo, „einzigen"Zug Salmanassars, nach Westen, bei dem er sich gegenüber der Koalition Hosec-Luli-Sib'e auf eine Partei in Israël und die von Sidon geführte Fronde gegen Tyrus stützen konnte.

Den ersten Zug Salmanassars gegen Hosea, von dem 2. K. 17 berichtet, wird man nun, da Menander aus tyrischen Quellen genau Entsprechendes über einen ersten Zug gegen Phönicien zu berichten weiss, wohl oder uibel als geschichtliche Thatsache hinnehmen müssen.

Ohnehin waren die Gründe, mit denen man ihn hinwegzubeweisen suchte, fadenscheinig genug. Man fragt, warum Salmanassar gegen Hosea gezogen sein sollte, da sich der Isrä̈literkönig doch bereits im Jahre $734 \mathrm{v}$. Chr. Tiglatpilesar dem Dritten zur Tributzahlung verpflichtet hatte. Dass aber bei erster Gelegenheit der Versuch gemacht wird, einer solche lästigen Verpflichtung ledig zu werden, ist doch nur natürlich, und zahllose Beispiele dafür finden sich in den assyrischen Königsinschriften. Auch Samaria hat ja bald nach der Eroberung durch Sargon versucht, das assyrische Joch abzuschütteln , ${ }^{1}$ ) obgleich hier die unmittelbare assyrische Verwaltung eingeführt und kein König vorhanden war, der den Aufstand organisiert hätte. Für den vorliegenden Fall brauchen wir uns mit solchen allgemeinen Erwägungen nicht zu begnügen. Mit höchster Wahrscheinlichkeit ist anzunehmen, dass Salmanassar IV. zu seinem ersten Zuge nach Westen (MenandelJosephos) mamentlich durch Nachrichten von Unterhandlungen, die mit Ägypten im Gange waren, veranlasst wurde. Salmanassar, der bei Lebzeiten seines Vaters den zu Assyrien als Provinz gehörigen Teil Phöniciens und Coelesyriens („die Provinz Simyra“) verwaltet hatte, hatte natürlich allerorten seine Verbindungen unter den Anhängern Assyriens, die den Hof von Niniveh über alle irgend verdächtige Vorgänge auf dem Laufenden hielten. Salmanassar griff in Phönicien ein, ehe die Verhandlungen mit Ägypten zum Abschluss gekommen waren. Und wenn wir im Jahre 724 sowohl Elulaios wie Hosea auf Agynten gestützt einen offenkundigen Versuch machen sehen, die assyrische Oberherrschaft abzuschütteln, so sind wir zu der Annahme geradezu gezwungen, dass auch an den vorbereitenden Verhandlungen mit Ägypten Hosea nicht unbeteiligt gewesen ist. Es müssen also die auf Störung der Verhandlungen und Vereitelung der Rüstungen zielenden Massnahmen des Assyrerkönigs, d. h. der erste Zug gegen Westen, wie Tyrus so auch Israël betroffen haben. Nur daraus, dass die früher angeknüpften

Vater in der Herrschaft Äthiopiens vorausgegangen oder gefolgt sein muss. Zu entscheiden, $o b$ in diesen Zusammenhang etwa doch die von Schaser, Ztschr. f. aeg. Sprache 23 , $1 \mathrm{ff}$, in die Zeit n a ch Sabako verwiesene Inschrift des Äthioperkönigs $I-s-p-r-w-t i$ gehört, in der es heisst: ,Du hast das Diadem Deines Bruders des Königs $X^{\dagger}$, muss weiterer Untersuchung vorbehalten bleiben.

1) Sargons Prunkinschr. 33. 
Verhandlungen nach dem Abzug Salmanassars (725) wieder aufgenommen werden konnten, erklärt es sich, dass sowohl Elulaios wie Hosea, die sich, weil sie überrumpelt worden waren, 725 gefügt hatten, bereits im folgenden Jahre wieder auf dem Platze waren.

So erhalten wir eine vollgen ïgende Erklärung von Salmanassars erstem Zuge gegen Hosea. Mit einem Lächeln der Erleichterung können wir die mit den grössten Unwahrscheinlichkeiten operierenden Versuche, ihn hinwegzudeuten, bei Seite legen. Während doch für die israëlitische Geschichte selbstverständlich die Israël betreffenden Kapitel der Königsbücher Hauptquelle sind, soll für diesen einen Fall das Juda behandelnde Kapitel, 2. K. 18., das nur von ein em Zuge weiss, das Richtige geben. Was der israëlitische Bericht mehr bietet, so u. a. auch die oben als völlig zutreffend erwiesene Gefangennahme Hoseas, sollen Zusätze sein, die auf Missverständnissen beruhen. Die auf Ausscheidung dieser Zusätze gerichteten Bemühungen, ${ }^{1}$ ) die niemals befriedigten, weil organische Bestandteile einer Nachricht sich eben nicht ausscheiden lassen, die niemals zum Ziel führenden Textesänderungen, zu denen man sich verstieg, - sie alle können wir jetzt auf sich beruhen lassen. $\left.{ }^{2}\right)$ Es handelt sich nicht um Zusätze im israëlitischen, sondern um Auslassungen und Ungenauigkeiten in dem an den Dingen weniger interessierten, in erster Linie Hiskia von Juda betreffenden Abschnitte.

Die Ergebnisse dieser Untersuchung sind kurz: 1. Menander und Jo:sephos berichten mit vollem Recht über einen zweimaligen $\mathrm{Zug}$ salmanassars IV. gegen Tyrus. Für Unternehmungen anderer Assyrerkönige, Tiglatpileser III., Sanherib, Assarhaddon, Assurbanabal, ist aus der Nachricht nichts zu entnehmen. 2. Die 5jährige, von Salmanassar $I V$. begonnene Belagerung währte von 724-720. Tyrus wurde durch Sargon II. so wenig erobert, wie durch spätere assyrische oder babylonische Könige. Die Belagerung wurde vielmehr, aufgehoben. 3. Lulk-Elulaios ist sowohl von Salmanassar IV. und Sargon II. wie von Sanherib bekämpft worden. Menanders Angabe, er habe 36 Jalıre regiert, ist un-

1) Diese Bemuhungen erinern im Verfahren und im Ergebnis an die Umdeutungen der Nachricht bei Josephos-Menander. Man lese z. B. Kittels Kommentar zu 2. Kön. $17 \mathrm{~S} .273 \mathrm{f}$, der sich grossenteils auf Winckuer, Alttest. Unters. $15 \mathrm{ff}$. stuitzt. Vers $5 \mathrm{ff}$. sollen nicht die Fortsetzung von V. 3 u. 4 sein, sondern ihnen parallel gehen. Vers 3 beziehe sicb auf Hoseas Aufstand gegen Tiglatpileser III. (ebenso Stade a. a. O. 600', s. dagegeu oben S. 470). Der ganze Abschnitt-Vers $3-6$ rede nur vom „König von Assur", der Name sei von einem Glossator aus 2. Kön. 18 hier eingetragen, "leider am unrechten Platz." Erst mit Vers 4 beginne die Zeit Salmanassars etc.

2) Gegen Wincklers und Benzingers Vorschläge mit Recht Krrter a. a. O.

2) Die chronologisehen Sehwierigkeiten hat schon TIELE (Bab.-ass. Gesch. I 237) geklärt: ,Ich vermute, dass das neunte Jahr sowie die Gefangennahme vor der Belagerung von dem Bibelschreiber richtig uberliefert wird, dass er aber irrtiumlich in dieses neunte Jahr die Einnahme der Stadt setzt.' Ist Hoseas 1. Jahr 733/2, so kann die, fruh im Jahre 724 erfolgte, Gefangennahme in dessen neuntes Jahr $725 / 4$ fallen. 


\section{C. F. Lehmann, Menander und Josephos über Salmanassar IV.}

anfechtbar: Dass Sanherib berichte, Luľ̂ sei nach seiner Flucht aus Sidon bereits im Jahre 701 oder kurz darnach gestorben, ist eine falsche Behauptung. Sanherib hat Sidon einen anderen König gegeben, der auch die mit Sidon verbündeten Städte beherrscht haben wird. Dagegen

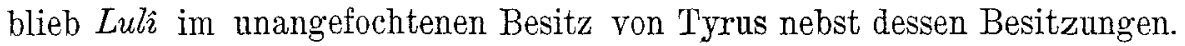
4. Unter Assarhaddon und Assurbanabal sind zwei getrennte Belagerungen von Tyrus zu verzeichnen, die beide erfolglos endeten. 5. Wie gegen Elulaios so ist Salmanassar $1 \mathrm{~V}$. zweimal gegen Hosea von Israël zu Felde gezogen, in beiden Fällen stand Hosea mit Tyrus und Ägypten im Bunde. Hosea ward zu Anfang des zweiten Feldzuges gefangen genommen.

Für Salmanassars Herrscherthätigkeit ergiebt sich uns nunmehr folgendes Bild: Bei Lebzeiten seines Vaters zum Statthalter der neueroberten Gebiete in Phönicien und im nördlichen Syrien ernannt (734), setzte er nach seiner Thronbesteigung (727) dessen Eroberungspolitik zielbewusst fort. Die Kenntnis der Verhältnisse des Westens und die Verbindungen, die er dort angeknüpft hatte, kamen ihm dabei zu Statten. So erhielt er rechtzeitige Kunde von einer von Tyrus und Israël geplanten Auflehnung gegen das Joch Assyriens, der er durch sein schnelles Erscheinen im Jahre 725 zuvorkam. Die bestehenden Verträge wurden erneuert, und Hosea wie Lulı verpflichteten sich zu weiterer Tributzahlung. Diese wurde dann im Jahre 724 auf weiteres Betreiben und im Vertrauen auf Ägypten verweigert und damit ein offenkundiger Kriegszustand eingeleitet. Salmanassars Operationen richteten sich auch jetzt wieder gegen Israël und Tyrus. Hosea wurde gefangen, Samaria widerstand. Durch einen Aufstand ans innerpolitischen Gründen gestïrzt und ums Leben gekommen , ${ }^{1}$ ) ging Salmanassar des Ruhmes, Samaria erobert zu haben, verlustig, brauchte aber auch nicht das Scheitern der Belagerung von Tyrus zu erleben. In der äussern Politik, über die wir allein näher informiert sind, erscheint er als des grossen Vaters nicht unebenbürtiger Sohn. ${ }^{2}$ )

1) S. den zu oben S. $126^{2}$ nachzutragenden Text K. 1349 (WINCKLER AOF. I $401 \mathrm{fi}$.), Z. 31

2) Nachtrag zu Teil I: Nach Veröffentlichung von Heft 1 dieses Bandes erhielt ich durch des Verfassers Gute Kenntnis von Eodard Merers Artikel Phoenicia in

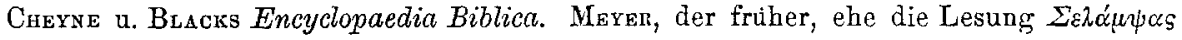
bekannt geworden war, die Josephos-Stelle auf Sanherib gedeutet hatte (GA. I $\$ 357 \mathrm{~A}$. u. 383 A.), bezieht jetzt den ersten Feldzug mit Menander auf Salmanassar IV., bleibt aber, unbewusst beeinflusst von der früheren Ansicht, auf halbem Wege stehen, indem

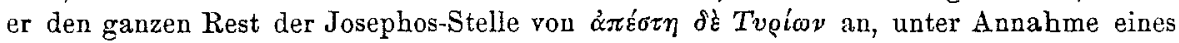
Irrtums des Menander, für Santerib in Anspruch nimmt. Meine obigen Ausführungen siud daher auch als gegen ED. Merens Ansicht gerichtet zu betrachten. 